\title{
A Study on Dynamic Domain Knowledge Visualized Navigation Mechanism
}

\author{
Wei Jiuhong Li Mengyang* Zhang Chunfei Duan YunNa Wang WeiHong
}

The Agricultural Division, JiLin University Changchun 130062 China

\begin{abstract}
We present an overall framework for dynamically visualized navigation mechanism of domain knowledge concerning learners' cognitive issues. The mechanism includes the construction of learning resource, domain knowledge entity, knowledge library based on domainentity, learners' model, visualization of knowledge library, and navigation system guided by domain-entity.
\end{abstract}

Keywords: knowledge visualization; navigation; learning resources

\section{Introduction}

Nowadays, domain knowledge distributing in the world wide scope is characterized by tremendous information capacity, comprehensive contents, and convenience facility. For example, MIT offers a website presenting all their open courseware for free usage by students and institutes around the world. The other university such as Lice and Tokyo also put forward their open course resources respectively. In China, Ministry of Education initiated the construction of selected courses library at university, provincial, and national levers respectively, and at same time, a uniform network course resources system was founded to provide abundant digitalized learning materials and equal opportunities for learners.
However, from the point of domain knowledge architecture there still exist three issues to be enhanced concerning about such open learning environment. A. the organization of course resources is mostly in form of single course or individual courseware, which is lack of the complete concepts overview and leads to weak integrity of the systematic domain knowledge. B. the way to access multiple course information varies with different discipline knowledge, and this causes unbalance between the huge knowledge architecture and the needs for learners to acquire special valuable piece of information. C. although the diversity of knowledge transferring media has enrich the content of domain knowledge, it raises the complexity to some extent for classifying and reorganizing course resources. Hence, it calls for an efficient navigation mechanism supported by visualization technologies which can present knowledge architecture rationally, and equip learners with powerful tools for rightly reconstructing, clearly memorizing, and properly applying the acquired domain knowledge.

\section{Related works}

The visualized knowledge navigation system can transform knowledge architecture into understandable and articulate concept map by exploring characteristics and associations of various disciplines. Eppler ${ }^{[1]}$ had reviewed couple of innovat-

\footnotetext{
* corresponding author.
} 
ing concepts coming from various kinds of disciplines, and illustrated how visualization mechanism played an activator and integrator role in cooperating study. Bresciani ${ }^{[2]}$ had investigated enterprise managers to address the visualization process for business planning, knowledge sharing, and assessment scheming, etc. they proposed visualization could promote cognition in many aspects. Nielsen ${ }^{[3]}$ considered navigation problems as answering the learners "where am I now? Where have I been? And where will I go?" The main task is to avoid navigation lost, and clear directing obstacles. Kboubi ${ }^{[4]}$ proposed a multiple-semantic navigation mechanism which consists of a domain-entity navigating method enabling learners explore information according to their special interest, a conceptassociation navigating method allowing for connotation search through conceptassociation map, and a resemblerelationship navigating method facilitating significance search by resembledocuments map. Zhanghuiping ${ }^{[5]}$ considered visualization technology can help domain knowledge construction by providing a kind of intuitionistic platform for intelligent interacting. In our study, the domain-entity navigating method is used to solve cognitive-payload problems and clear lost-direction obstacle.

\section{The main mechanism}

\section{1. construction of learning resources}

Learning resources coming from Website, scientific literature, traditional documents, and databases need to be collected, analyzed, and coordinated according to requirements of learners to form a brief indexing database. Then, conforming to every discipline theory framework, the key literature and knowledge structures are analyzed. The website resources are classified by information units and levels. Therefore, we can determine the contents of each knowledge module, the knowledge media such as journals, thesis, and specialists. These source data can form an objective document for perspective uses.

\section{2. construction of knowledge entity based on discipline formulations}

The domain knowledge can be formulated to various layers by investigating the knowledge entity concepts, and verify the hierarchy structures such as filiations, fatalities, innovations, and sub-classes. Based on five kinds of module operators, the semantic logic of domain knowledge can be described. By presenting the RDF frameworks of domain knowledge, we can analyze the entity and achieve the exact semantic OWL description. The knowledge engineers and domain specialists can use this modeling tool to describe the appropriate scientific literature, to construct the class and attribute levels, and finally the knowledge entity. This project has referenced model like "sevensteps" developed by Stanford University, i.e. via requisite semantics and analysis, we have got a sound ground for knowledge visualization.

\section{3. construction of knowledge base built upon domain entity}

Having upon basis of domain knowledge entity, we can now further the entity assessments and evolution which are a looping process concerning about construction issues during entity analysis. The evaluations of entity will emphasis the consistency, maturity, and intelligence issues. If the expectation is achieved, the entity evolution process begins, otherwise, we should take advantages of domain specialists and knowledge engineers combination to check the system limitations, and return to the structure analysis once again until 
our objective are fulfilled. The knowledge entity evolution process is in fact the adjusting process for proper classes, attributes, and concepts, then updates dynamically by certain techniques and methods according to various domain demands. The entity assessment and evolution process can consummate to a satisfied domain knowledge base. And during the construction process, the evolution itself guarantee the dynamically updating of classes, attributes and relations information then enable the knowledge nodes and links have fitness to learning environments, and these measures put a background for visualize navigation of domain knowledge.

\section{4. construction the model of learn- ers}

Generally, learners have various interests and cognitive types in certain domain knowledge. The learner model then should present all these different aspects especially the acquisition, formulating, storage and modifying learner's interests and all of the cognitive issues. Further, this information also need reasoning to classify and recognize various types of learning model, so as to draw a proper conclusion about learners' requirements and learning task. This project will explore learners' information from three aspects: A. explore learners browsing history such as domain introduction, specialists, scientific literatures, reading times, reading frequency, time consuming and so on. B. explore learners attitude towards recommended knowledge resources such as "like, dislike, not known, or adopt, save, and copy" etc. C. explores learners' keywords in searching some certain website engine to track learners' learning records. We can also set different weight on above three aspects in order to evaluate learners' interests and learning characteristics.

\section{5. visualization of domain knowledge base}

In this item, we have visualized the domain knowledge entity to fit learner's cognitive characteristics and the knowledge nodes, knowledge links, knowledge associations are all presented in a intuitionistic way. The system is designed under such considerations: domain knowledge types, reasons for taking visualization technology, formulation of the visualization, and the purposes of visualization. The main functions include exploring learner's interests and cognitive characteristic, dynamically presenting learning object (domain knowledge, resources, learner's interests model, using pearl eye view, concept map, arbor model to explore knowledge evolution and differentiation process, knowledge engineering, domain specialists and learners all can add or remove some domain knowledge target, sharing, and evolution of the system. Learner can use standard electrical documents, adding website links, videos, graphics, and books. And according to the learner's requirements any interesting documents or refreshing information can be added up into the system. The knowledge recommendations process and visualization process both need the knowledge objects to be dynamically reconstructed, and this leads to a iterative, coordinating arrangement.

\section{6. navigation system guided by do- main knowledge entity}

The navigation system use information about learner's individual interest and cognitive character to set the start point of learning process. Knowledge nodes are selected by learners actively, so as to promote learner's exploring ability. In this kind of navigation system, learners can search certain topics which they are interested in, and the knowledge base is presented semantically in a visualized 
fashion. Main function of this module includes retrieving knowledge items and resources via a concept searching, then by organizing the topic relevance, different shape or color will display various level of knowledge nodes and links. In such a visualized manner, learners can explore every knowledge items one by one. The navigation system offers a knowledge presentation according to individual learner's cognitive model to avoid getting lost in exploring process and enable learners to locate their knowledge nodes directly.

\section{7. system deployment}

Our navigation mechanism is implemented using helix software development process. The system collects most of open course resources and navigates in a dynamically visualizing mechanism on purposes of improving learner's achievement in an open resource leaning environment. After the system requirement analysis process, we set up four sub-module including the dynamic visualizing navigation module, resources aggregation module, learning behavior statistics module, and interactive comment module. We also redesign the system user interface in order to fit learner's common habits.

\section{8. demonstration}

To check the effectiveness of the dynamical visualized navigation mechanism, we have investigated user's satisfaction ratio by questionnaire, learner's assertiveness degree by technology receive model, and we have analyzed learner's learning behavior by using statistical measures. Finally, we investigate the sore of learner's final examination to evaluate to what extent this mechanism improve learner's achievements.

\section{Conclusion}

We aim to study the key factor in visualized navigation system including its objective, paradigm, and strategy. By merging the mechanism of individualized recommendation and visualized navigation, learners can ameliorate their work-flows in studying process so as to fit their individual interests, studying habits, and therefore, to get the maximum accomplishment in studying.

\section{References}

[1] M.J. Eppler \& R.A. Burkhard, "Knowledge Visualization," Encyclopedia of Knowledge Management, 2011, pp. 987-999.

[2] S. Bresciani \& M.J. Eppler, "Choosing Knowledge Visualizations to Augment Cognition: the Managers' View," IEEE Proceedings of the International Conference on Information Visualization, London, 2010.

[3] J. Nielsen, "Designing the Web Usability", Indianapolis: New Riders Publishing, 2000.

[4] F. Kboubi, A. Chaibi, M. BenAhmed, "Semantic Visualization and Navigation in Textual Corpus", International Journal of Information Sciences and Techniques(IJIST), Vol.2, No.1, January 2012,pp53-63.

[5] Zhang JuiPing, "Study on knowledge construction based on visualization technology", information theory and Practice, No.10,2010,pp101-104. 